\title{
Energy Dissipation based Longitudinal and Lateral Coupling Control for Intelligent Vehicles
}

\author{
Rui Zhang, Yulin Ma, IEEE Member, Zhixiong Li, IEEE Member, Reza Malekian, IEEE Senior \\ Member, Miguel Angel Sotelo, IEEE Senior Member
}

\begin{abstract}
This paper proposes a combined longitudinal and lateral control approach for an intelligent vehicle system based on energy dissipation. The vehicle system dynamics resembles a series of mass/spring/damper systems that are dissipative, i.e., the energy of the system decays to zero eventually. Thus, the nonlinear-optimal longitudinal and lateral coupling control problem of the intelligent vehicle system is transformed into a dissipative control design based on an energy storage function. To satisfy the $\gamma$-performance, with respect to the quadratic supply rate, the storage function is developed by using a back-stepping based Lyapunov method and a step-by-step improvement of performance bounds. A dissipative feedback control law is formulated by successive approximation based on the step-by-step reduction of the value of $\gamma$. The results of the adaptive vehicle control simulations and test-bed experiments are provided and verified by the respective comparison of energy consumption on different values of $\gamma$ and speed adaption under different road geometries.
\end{abstract}

\section{INTRODUCTION}

Intelligent vehicle (IV) systems are attracting increasing attention from a technological and commercial point of view ${ }^{[1,}$ 2]. The unmanned vehicle program has been recognized as one of the hottest topics ${ }^{[3]}$. Typical researches and applications of unmanned vehicle program can be found in the Autopilot

* Research supported in part by the National Key Research and Development Program (2016YFB0100903), and in part by the National Natural Science Foundation of China (61503284, 51505475 and 51408417) and Yingcai Project of CUMT (YC170001).

Dr. R. Zhang is with School of Automotive and Transportation, Tianjin University of Technology and Education, Tianjin, 300222 China (e-mail: zhangrui@tute.edu.cn).

Dr. Y. Ma is an associate professor with National Center of ITS Engineering and Technology, Research Institute of Highway, Ministry of Transport, Beijing, 100088 China (corresponding author e-mail: myl@itsc.cn).

Dr. Z. Li is with School of Mechatronic Engineering, China University of Mining and Technology, Xuzhou 221000, China; he is also with Department of Mechanical Engineering, Iowa State University, Ames 50010, USA (corresponding author e-mail: zhixiong.li@ieee.org).

Dr. R. Malekian is an associate professor with Department of Electrical, Electronic \& Computer Engineering, University of Pretoria, Pretoria 0002, South Africa (email: reza.malekian@ieee.org).

Prof. M. Sotelo is a Professor at the Department of Computer Engineering. University of Alcalá, Alcalá de Henares (Madrid), Spain (email: miguel.sotelo@uah.es) system by Tesla Motors. Google, Uber and GM are also contributing great efforts on unmanned vehicle programs. In fact, intelligent vehicles (IVs)/autonomous vehicles (UVs) have been regarded as the sixth disruptive technology, and in a close future, IVs/UVs will coexist with conventional vehicles in one's daily life ${ }^{[4]}$.

Advanced driver assistance system (ADAS) is the most important component/development for intelligent vehicles ${ }^{[5]}$. ADAS involves with three key techniques, vehicle sensing, vehicle position system, and intelligent control, to achieve unmanned control for IVs. Some ADAS systems has been studied for the purposes of collision avoidance and platooning assistance ${ }^{[6,7]}$, side slip and rollover avoidance ${ }^{[8]}$, and lane keeping assistance ${ }^{[9]}$. Especially, in the platooning control, Guo and Yue ${ }^{[10,11]}$ presented guaranteed-cost controller considering the effect of sensing range limitation and sensor failure, yield a useful string stable platoon control algorithm. $\mathrm{Li}$ et al ${ }^{[12]}$ presented multiple- model switching control for platoon level automation. The switching control system automatically selects the most appropriate candidate controller into the loop, according to the errors between current vehicle dynamics and multiple models. Xia et al ${ }^{[13]}$ presents a decoupling method of synthesis of distributed $\mathrm{H}_{\infty}$ controller for heterogeneous vehicular platoons with complex interaction topologies. Based on this almost decoupled system, the distributed $\mathrm{H}_{\infty}$ controller can balance the performances of robustness and disturbance attenuation ability, which is a good idea for the optimization of control performance. Among existing researches, the longitudinal and lateral control, also known as adaptive cruise control (ACC) and steering control ${ }^{[14]}$, are two of the most conventional forms of ADAS. How to realize unmanned control of ACC or/and steering wheel of a vehicle remains one challenging task facing researchers in this area ${ }^{[15,16]}$. Zhang and Ioannou ${ }^{[17]}$ put forward a PID-based ACC control method with nonlinear filter, designed the optimal vehicle speed trajectory under different vehicle spacing strategy, and improved the fuel economy by reducing the gap between the vehicles. Corona et al. ${ }^{[18]}$ proposed a predictive ACC control method. The cost function mainly considered the tracking error, and took the acceleration and the minimum distance as the constraint condition, to guarantee the comfort and the safety of the vehicle while realizing the automatic vehicle-following control. Hosseinnia et al. ${ }^{[15]}$ designed the hybrid fractional-order ACC control method for an automatic vehicle. The experimental test demonstrated good distance-follow performance of the proposed hybrid ACC controller. Onieva et al. ${ }^{[16]}$ presented a fuzzy controller for steering control. The genetic algorithm was employed to optimize the Fuzzy controllers to guarantee automatic driving 
of an unmanned car. Velhal and Thomas ${ }^{[19]}$ improved the linear time varying model for steering control of an autonomous vehicle. Although these researches have contributed to excellent insight and knowledge to the longitudinal and lateral control for autonomous vehicles, they limit themselves to separate the longitudinal control from lateral control; vice versa. Very limited work has been done to combine the longitudinal and lateral control into a generic framework ${ }^{[20-22]}$.

In order to address the combined longitudinal and lateral control, the state-space representation of the IV dynamic system is adopted to build the multiple-input multiple-output (MIMO) control models ${ }^{[20-24]}$. However, the implementation of these MIMO controllers (e.g., prediction control ${ }^{[20]}$ or $\mathrm{H}_{\infty}$ control ${ }^{[25]}$ ) often imposes offline computation and strict constraints in both simulations and experiments. For example, when forming the Hamilton function in the $\mathrm{H}_{\infty}$ control, the feedback factor is often formulated by using Riccati equation or algebraic iteration ${ }^{[25]}$, which increase the offline computation. In addition, the control inputs need to be weighted for the MIMO controllers and the convergence of iterative approximate solution is not always guaranteed.

The work described in this paper has been carried out at the National Key Research and Development Program at the National Center of ITS Engineering and Technology, a part of the Ministry of Transport of China for ITS Research. More concretely, the aim of the present work is the longitudinal and lateral coupling control for a mass-produced vehicle. A dissipative control approach is proposed for an IV longitudinal and lateral coupling system. The benefits of the proposed approach as opposed to existing popular ones is that it does not require solving Riccati equations or Hamilton-Jaccobi-Issacs (HJI) inequalities ${ }^{[26]}$. So, it is convenient and efficient for improving the control performance. To the best of our knowledge, the combined longitudinal and lateral control using energy dissipation has not been found yet. The effectiveness of the proposed dissipative control approach is verified by both simulation and experimental tests.

The rest of this paper is organized as follows. Section II presents a longitudinal and lateral coupling vehicle model and the problem statement. The dissipative control law is designed in Section III, where the procedures for energy storage function development and dissipative control optimization are detailed. In Section IV, experimental analysis is used to demonstrate the efficiency of the approach. The paper is concluded in Section V.

\section{II.LONGITUDINAL AND LATERAL COUPLING VEHICLE MODEL AND PROBLEM STATEMENT}

\section{A. A dissipative system with the quadratic supply rate}

A dissipative system is characterized by the following property: at any time, the amount of energy that the system can potentially supply to its environment cannot exceed the amount of energy the system receives from the environment. In other words, as time evolves, a dissipative system absorbs a fraction of its supplied energy and transforms the rest into energy 'losses', such as heat, an increase of entropy, and electro-magnetic radiation ${ }^{[27,28]}$. An IV system can be identified as being dissipative because it interacts with the environment by a loss of energy due to friction, disturbance, and mechanical losses. Hence, it is reasonable to apply the energy dissipation analysis to the IV systems.

Consider the generic state-space equation of a dissipative system in Eq. (1):

$$
\left\{\begin{array}{l}
\dot{x}=f(x)+g(x) u \\
y=h(x)
\end{array}\right.
$$

where $x \in X \subset \mathbf{R}^{n}, u \in U \subset \mathbf{R}^{p}$ are the state variable and the control input variable, respectively and $y \in Y \subset \mathbf{R}^{m}$ is the output vector. Because Eq. (1) describes the generic equation of a dissipative system, the concrete form of the state variable $x$ can be flexible based on specific case study. For instance, $x$ can choose the velocity or displacement of the dissipative system, corresponding to a first order derivative or a second order derivative in the concrete state-space equation, respectively. In this Section, we discuss the properties of a dissipative system using its generic state-space equation in Eq. (1). Following [27], we obtain the following definitions.

Definition 1: Let $\gamma \geq 0$. System in Eq. (1) is said to have $L_{2}$-gain less than or equal to $\gamma$ if

$$
\int_{0}^{T}\|y(t)\|^{2} \mathrm{~d} t \leq \gamma^{2} \int_{0}^{T}\|u(t)\|^{2} \mathrm{~d} t
$$

for all $T \geq 0$ and all $u \in L_{2}(0, T)$, with $y(t)=h\left(\varphi\left(t, 0, x_{0}, u\right)\right)$ denoting the output of Eq. (1) resulting from $u$ for initial state $x(0)=x_{0}$. The system has $L_{2}$-gain $<\gamma$ if there exists some 0 $\leq \widetilde{\gamma}<\gamma$ such that Eq. (2) holds for $\widetilde{\gamma}$.

Remark: Definition 1 is a special case of the general definition of dissipativity as given in [28], where, instead of the supply rate $s(u, y)=\gamma^{2}\|u\|^{2}-\|y\|^{2}$ as used in Definition 1, an arbitrary supply rate $s(u, y)$ is being considered.

Notice that condition in Eq. (2) can be equivalently expressed as

$$
\lim _{T \rightarrow \infty} \inf _{\substack{u \in L_{2}(0, T) \\ x(0)=x_{0}}} \int_{0}^{T}\left(\gamma^{2}\|u(t)\|^{2}-\|y(t)\|^{2}\right) \mathrm{d} t \geq 0
$$

According to theorem 2 in [29], there exists a smooth solution $S \geq 0$ of the dissipation inequality

$$
\frac{\partial S}{\partial x} f(x)+\frac{\partial S}{\partial x} g(x) u \leq \frac{1}{2}\left(\gamma^{2}\|u\|^{2}-\|y\|^{2}\right) \quad S\left(x_{0}\right)=0
$$

where the function $S(x)$ is called the energy storage function.

\section{B. Longitudinal and lateral coupling vehicle model}

Due to the coupling effect between longitudinal and lateral motion of IV, a three-degree of freedom (DOF) vehicle model that includes longitudinal motion, lateral motion and yaw motion is always used in the analysis. In order to simplify the model derivation and the computational burden, it is assumed that roll, pitch, and bounce motions are negligible, the effect of suspension on the tire axels is also negligible, and brake, throttle, and steering dynamics are discounted. Thus, in this specific research objective, the displacement is used to establish the combined longitudinal and lateral IV model with 3 DOFs, which can be expressed in the following second order derivative form ${ }^{[23,24]}$ 


$$
\left\{\begin{array}{c}
\ddot{\tilde{x}}=a_{1} \dot{\tilde{x}}^{2}+\tilde{y} \dot{\psi}+C_{f} \frac{\tilde{y}+l_{f} \dot{\psi}}{m \dot{\tilde{x}}} \delta+\frac{F-\mu m g}{m} \\
\ddot{\tilde{y}}=-a_{2} \frac{\dot{\tilde{y}}}{\dot{\tilde{x}}}-\left(\dot{\tilde{x}}+\frac{k a_{3}}{\dot{\tilde{x}}}\right) \dot{\psi}+\frac{F-\mu \lambda\left(m g-k_{L} \dot{\tilde{x}}^{2}\right)+C_{f}}{m} \delta \\
\ddot{\psi}=-a_{4} \frac{\dot{\psi}}{\dot{\tilde{x}}}-a_{3} \frac{\tilde{y}}{\dot{\tilde{x}}}+\frac{l_{f}}{I_{z}} \frac{F-\mu \lambda\left(m g-k_{L} \dot{\tilde{x}}^{2}\right)+C_{f}}{m} \delta
\end{array}\right.
$$

where $\tilde{x}$ denotes the longitudinal displacement, $\tilde{y}$ denotes the lateral displacement, $\psi$ denotes the yaw angle, and $F$ and $\delta$ are the traction force and steering angle, respectively. The vehicle parameters are shown in Table 1 below.

TABLE I. VEHICLE PARAMETERS

\begin{tabular}{cc||cc}
\hline Parameters & Definition & Parameters & Definition \\
\hline $\mathrm{m} / \mathrm{kg}$ & Mass & $C_{r} / \mathrm{N} \cdot \mathrm{rad}^{-1}$ & $\begin{array}{c}\text { Cornering } \\
\text { stiffness-rear } \\
\text { Aerodynamics-drag } \\
\text { coefficient }\end{array}$ \\
$I_{f} / \mathrm{kg} \cdot \mathrm{m}^{2}$ & $\begin{array}{c}\text { Yaw inertia } \\
\text { Distance-front } \\
\text { axle to c.o.g }\end{array}$ & $k_{D} / \mathrm{N} \cdot \mathrm{s}^{2} \cdot \mathrm{m}^{-2}$ & $\begin{array}{c}\text { Aerodynamics-lift } \\
\text { coefficient }\end{array}$ \\
$l_{r} / \mathrm{m}$ & $\begin{array}{c}\text { Distance-rear } \\
\text { axle to c.o.g } \\
\text { Cornering }\end{array}$ & $\mu$ & $\begin{array}{c}\text { Rolling resistance } \\
\text { coefficient }\end{array}$ \\
$C_{f} / \mathrm{N} \cdot \mathrm{rad}^{-1}$ & stiffness-front & $\mathrm{g} / \mathrm{m} \cdot \mathrm{s}^{-2}$ & $\begin{array}{c}\text { Acceleration of } \\
\text { gravity }\end{array}$ \\
\hline
\end{tabular}

$$
\left\{\begin{array}{l}
a_{1}=\frac{\mu k_{L}-k_{D}}{m}, a_{2}=\frac{\left(C_{f}+C_{r}\right)}{m}, a_{3}=\frac{\left(C_{f} l_{f}-C_{r} l_{r}\right)}{I_{z}}, a_{4}=\frac{\left(C_{f} l_{f}^{2}+C_{r} l_{r}^{2}\right)}{I_{z}} \\
\lambda=\frac{l_{r}}{l_{f}+l_{r}}, k=\frac{I_{z}}{m}
\end{array}\right.
$$

To verify the formulated control laws, we make transformation for inputs of the IV model as

$$
\left\{\begin{array}{l}
\ddot{\tilde{x}}=a_{1} \dot{\tilde{x}}^{2}+\dot{\tilde{y}} \dot{\psi}+u_{1} \\
\ddot{\tilde{y}}=-a_{2} \frac{\dot{\tilde{y}}}{\dot{\tilde{x}}}-\left(\dot{\tilde{x}}+\frac{k a_{3}}{\dot{\tilde{x}}}\right) \dot{\psi}+u_{2} \\
\ddot{\psi}=-a_{4} \frac{\dot{\psi}}{\dot{\tilde{x}}}-a_{3} \frac{\dot{\tilde{y}}}{\dot{\tilde{x}}}+\frac{m l_{f}}{I_{z}} u_{2}
\end{array}\right.
$$

where

$$
\left\{\begin{array}{l}
u_{1}=C_{f} \frac{\dot{\tilde{y}}+l_{f} \dot{\psi}}{m \tilde{\tilde{x}}} \delta+\frac{F-\mu m g}{m} \\
u_{2}=\frac{F-\mu \lambda\left(m g-k_{L} \dot{\tilde{x}}^{2}\right)+C_{f}}{m} \delta
\end{array}\right.
$$

Because the displacement and yaw angle of the IV model is adopted as the basic state variables, the state-space equation of the vehicle model in Eq. (6) can be rewritten in a second order derivative form in this specific case study by Eq. (7)

$$
\left\{\begin{array}{l}
\ddot{\mathbf{x}}=f(\mathbf{x}) \dot{\mathbf{x}}+g_{1}(\mathbf{x}) \mathbf{d}+g_{2}(\mathbf{x}) \mathbf{u} \\
\mathbf{z}=h(\mathbf{x})
\end{array}\right.
$$

where $\mathbf{x}=[\tilde{x}, \tilde{y}, \psi]^{\mathrm{T}}$ are the state variables and $\dot{\mathbf{x}}=\left[v_{x}\right.$, $\left.v_{y}, \dot{\psi}\right]^{\mathrm{T}}$, where $v_{x}$ and $v_{y}$ are the longitudinal and lateral velocities; $\mathbf{d}=\left[d_{1}, d_{2}\right]^{\mathrm{T}}$ are the disturbances on the vehicle longitudinal and lateral dynamics, respectively; $\mathbf{u}=\left[u_{1}, u_{2}\right]^{\mathrm{T}}$ are the control laws of vehicle longitudinal and lateral dynamics; the cost function $\mathbf{z}$, which is given by a general expression $h(\mathbf{x})$, can be decomposed into two components (i.e., $z_{1}$ and $z_{2}$ ) along the vehicle longitudinal and lateral directions. The functions in the state transition equation in Eq. (7) can be expressed as

$$
f(\mathbf{x})=\left[\begin{array}{ccc}
a_{1} \dot{x} & \dot{\psi} & 0 \\
-\dot{\psi} & -\frac{a_{2}}{\dot{x}} & -\frac{k a_{3}}{\dot{x}} \\
0 & -\frac{a_{3}}{\dot{x}} & -\frac{a_{4}}{\dot{x}}
\end{array}\right], g_{1}(\mathbf{x})=\left[\begin{array}{cc}
1 & 0 \\
0 & 1 \\
0 & 1
\end{array}\right], g_{2}(\mathbf{x})=\left[\begin{array}{cc}
1 & 0 \\
0 & 1 \\
0 & \frac{m l_{f}}{I_{z}}
\end{array}\right]
$$

Here, although the system disturbance $\mathbf{d}$ is unknown, it can be always decomposed into two components (i.e., $d_{1}$ and $d_{2}$ ) along the vehicle longitudinal and lateral directions. The goal of energy dissipation control is to meet the $\gamma$-performance criteria between $\mathbf{d}$ and $\mathbf{z}$ [27]. A suitable $\mathbf{u}$ is required to control the system dynamic behavior by eliminating the control error between desired and actual outputs of the system. Generally, $\mathbf{u}$ is the controller output, so it is determined by the designed control law of the controller according to the control error and can be independent from $\mathbf{d}$ in Eq. (7). To achieve the quadratic dissipative $\gamma$-performance from the disturbance $\mathbf{d}$ to the cost function $\mathbf{z}$ in the whole closed loop system, a combined longitudinal and lateral control dissipative controller can be designed by means of developing the energy storage function. So, the combined longitudinal and lateral dissipative control problem can be described as:

1) For the longitudinal dynamics, we choose $u_{1}$ to make the longitudinal control system meet the following conditions:

- When $d_{1}=0$, the control system will be asymptotically stable if the longitudinal tracking error between the actual velocity and the desired one converges to zero, i.e., $\lim _{t \rightarrow \infty}\left|v_{x}-v_{x, \mathrm{~d}}\right|=0$, for the desired longitudinal velocity $v_{x, \mathrm{~d}}$

- For any $T>0$, the $\gamma$-performance criteria can be ensured, i.e.,

$$
\int_{0}^{T}\left\|z_{1}(t)\right\|^{2} \mathrm{~d} t \leq \gamma^{2} \int_{0}^{T}\left\|d_{1}(t)\right\|^{2} \mathrm{~d} t, \quad \forall d_{1} \in L_{2}[0, T]
$$

2) For the lateral dynamics, we also choose $u_{2}$ to make the lateral control system meet the following conditions:

- When $d_{2}=0$, the control system will be asymptotically stable if both lateral tracking error and yaw tracking error converges to zero, i.e.,

$\lim _{t \rightarrow \infty}\left|v_{y}-v_{y, \mathrm{~d}}\right|=0$, for the desired lateral velocity $v_{y, \mathrm{~d}}$, and $\lim _{t \rightarrow \infty}\left|\dot{\psi}-\dot{\psi}_{\mathrm{d}}\right|=0$, for the desired yaw rate $\dot{\psi}_{d}$

- For any $T>0$, the $\gamma$-performance criteria can be ensured, i.e.,

$$
\int_{0}^{T}\left\|z_{2}(t)\right\|^{2} \mathrm{~d} t \leq \gamma^{2} \int_{0}^{T}\left\|d_{2}(t)\right\|^{2} \mathrm{~d} t, \quad \forall d_{2} \in L_{2}[0, T]
$$




\section{DISSIPATIVE CONTROL LAWS FORMULATION}

Let us look back at the dissipation inequality (4). It is well known that if the storage function $S(x)$ is smooth and differentiable, the differential dissipation inequality will be

$$
\left.\dot{S}(x)\right|_{(4)} \leq \frac{1}{2}\left(\gamma^{2}\|u(t)\|_{U}^{2}-\|y(t)\|_{Y}^{2}\right), \quad \forall T \geq 0
$$

As the storage function can be taken as the Lyapunov function, a positive definite Lyapunov-like function $V(\mathbf{x})$ can be defined for Eq. (7) to meet the $\gamma$-performance criteria:

$$
\dot{V}(\mathbf{x})=\dot{S}(\mathbf{x}) \leq \frac{1}{2}\left(\gamma^{2}\|\mathbf{d}\|^{2}-\|\mathbf{z}\|^{2}\right)
$$

A back-stepping method is usually used to develop the Lyapunov function. The basic idea of the back-stepping method is to select recursively appropriate functions of the state variables as pseudo-control inputs for lower dimension subsystems of the overall system ${ }^{[30,31]}$. Each back-stepping stage results in a new pseudo-control design, expressed in terms of the pseudo-control design from the preceding stages. The procedure terminates a feedback design for the true control input that achieves the original design objective by virtue of the final Lyapunov function. This final function is formed by summing the Lyapunov functions associated with individual design stages. Here the energy storage function of the system in Eq. (7) is expressed as:

$$
\left\{\begin{array}{l}
V_{\text {long }}=\lambda_{1}\left[\frac{1}{2} d_{1}(t)\right]^{2}+\lambda_{2}\left[\frac{1}{2}\left(\dot{x}_{1}-\dot{x}_{1, \mathrm{~d}}\right)^{2}\right]+\lambda_{3}\left[\frac{1}{2}\left(x_{1}-x_{1, \mathrm{~d}}\right)^{2}\right] \\
V_{\text {lat }}=\lambda_{1}\left[\frac{1}{2} d_{2}(t)\right]^{2}+\lambda_{2}\left[\frac{1}{2}\left(\dot{x}_{2}-\dot{x}_{2, \mathrm{~d}}\right)^{2}\right]+\lambda_{3}\left[\frac{1}{2}\left(x_{2}-x_{2, \mathrm{~d}}\right)^{2}\right]
\end{array}\right.
$$

where $V_{\text {long }}$ and $V_{\text {lat }}$ are the storage functions of the longitudinal and lateral control subsystems, respectively; $\lambda_{1}, \lambda_{2}$ and $\lambda_{3}$ are the constant weights that are chosen in such a way that the typical magnitudes of the cost terms achieve a good balance between the cost function terms; $x_{1}$ and $x_{2}(=\tilde{x}$ and $\tilde{y})$ are respectively the longitudinal and lateral displacements and $x_{1, \mathrm{~d}}$ and $x_{2, \mathrm{~d}}$ are the desired longitudinal and lateral displacements, respectively. Here, let $x_{3}=\psi$ and $x_{3, \mathrm{~d}}$ is the desired yaw angle, then $x_{3, \mathrm{~d}}$ can be calculated by [32]

$$
\left\{\begin{array}{c}
x_{3, \mathrm{~d}}=\arctan \frac{\dot{x}_{2}}{\dot{x}_{1}} \\
\dot{x}_{3, \mathrm{~d}}=\frac{\ddot{x}_{2} \dot{x}_{1}-\dot{x}_{2} \ddot{x}_{1}}{\dot{x}_{1}^{2}+\dot{x}_{2}^{2}} \\
\ddot{x}_{3, \mathrm{~d}}=\frac{\left\{\left(\dddot{x}_{2} \dot{x}_{1}-\dot{x}_{2} \dddot{x}_{1}\right)\left(\dot{x}_{1}^{2}+\dot{x}_{2}^{2}\right)-2 \ddot{x}_{1} \ddot{x}_{2}\left(\dot{x}_{1}^{2}-\dot{x}_{2}^{2}\right)+2 \dot{x}_{1} \dot{x}_{2}\left(\ddot{x}_{1}^{2}-\ddot{x}_{2}^{2}\right)\right\}}{\left(\dot{x}_{1}^{2}+\dot{x}_{2}^{2}\right)^{2}}
\end{array}\right.
$$

The procedure of the dissipative control law formulation is discussed below.

In terms of the longitudinal control subsystem, a sliding surface $s_{1}$ is taken as the cost function $z_{1}$

$$
z_{1}=s_{1}=c_{1} e_{1}+\dot{e}_{1}
$$

where $c_{1}\left(c_{1}>0\right)$ is a constant, and

$$
\left\{\begin{array}{l}
e_{1}=x_{1}-x_{1, \mathrm{~d}} \\
\dot{e}_{1}=\dot{x}_{1}-\dot{x}_{1, \mathrm{~d}}
\end{array}\right.
$$

Putting (11) into the longitudinal storage function of (10), yielding

$$
V_{\text {long }}=\lambda_{1}\left[\frac{1}{2} d_{1}^{2}\right]+\lambda_{2}\left[\frac{1}{2} \dot{e}_{1}^{2}\right]+\lambda_{3}\left[\frac{1}{2} e_{1}^{2}\right]
$$

According to Eq. (7), the derivative of $V_{\text {long }}$ can be expressed as

$$
\begin{aligned}
\dot{V}_{\text {long }} & =\lambda_{1} d_{1} \dot{d}_{1}+\lambda_{2} \dot{e}_{1} \ddot{e}_{1}+\lambda_{3} e_{1} \dot{e}_{1} \\
& =\lambda_{1} d_{1} \dot{d}_{1}+\lambda_{2} \dot{e}_{1}\left(a_{1} v_{x}^{2}+v_{y} \dot{\psi}+u_{1}-\ddot{x}_{1, \mathrm{~d}}\right)+\lambda_{3} e_{1} \dot{e}_{1} \\
& =\lambda_{1} d_{1} \dot{d}_{1}+\lambda_{2} \dot{e}_{1}\left(a_{1} v_{x}^{2}+v_{y} \dot{\psi}\right)+\lambda_{2} \dot{e}_{1}\left(u_{1}-\ddot{x}_{1, \mathrm{~d}}\right)+\lambda_{3} e_{1} \dot{e}_{1}
\end{aligned}
$$

In order to satisfying (9), we define the disturbances of vehicle longitudinal dynamics as

$$
d_{1}(t)=\int_{0}^{t}\left(a_{1} v_{x}^{2}+v_{y} \dot{\psi}\right) e_{1} \mathrm{~d} t
$$

and the longitudinal control law $u_{1}$ is

$$
u_{1}=-\lambda_{1} c_{1} e_{1}-\lambda_{3} \dot{e}_{1}-\frac{1}{2 \gamma^{2}} s_{1}^{2}+\ddot{x}_{1, \mathrm{~d}}
$$

The proof of the control law $u_{1}$ is obtained as follows. Firstly, Putting (14), (15) into (13), yielding

$$
\begin{aligned}
\dot{V}_{\text {long }} & =\lambda_{1} d_{1}\left(a_{1} v_{x}^{2}+v_{y} \dot{\psi}\right) e_{1}+\lambda_{2} \dot{e}_{1}\left(a_{1} v_{x}^{2}+v_{y} \dot{\psi}\right)+\lambda_{2} \dot{e}_{1}\left(-\lambda_{1} c_{1} e_{1}-\lambda_{3} \dot{e}_{1}-\frac{1}{2 \gamma^{2}} s_{1}^{2}\right)+\lambda_{3} e_{1} \dot{e}_{1} \\
& =\lambda_{2}\left(\frac{\lambda_{1} d_{1}}{\lambda_{2}} e_{1}+\dot{e}_{1}\right)\left(a_{1} v_{x}^{2}+v_{y} \dot{\psi}\right)-\left(\lambda_{2} \lambda_{1} c_{1}-\lambda_{3}\right) \dot{e}_{1} e_{1}-\lambda_{2} \lambda_{3} \dot{e}_{1}^{2}-\frac{1}{2 \gamma^{2}} \lambda_{2} s_{1}^{2} \dot{e}_{1} \\
& \leq \frac{-c_{1} \lambda_{1} d_{1} e_{1}}{\lambda_{2}\left(a_{1} v_{x}^{2}+v_{y} \dot{\psi}\right)}+\lambda_{2} s_{1} d_{1}-\left[4 e_{1}^{2}+4 e_{1} \dot{e}_{1}+\dot{e}_{1}^{2}\right]+4 e_{1}^{2}-\frac{1}{2 \gamma^{2}} s_{1}^{2} \\
& \leq-\frac{c_{1} \lambda_{1}}{\lambda_{2}} e_{1}^{2}+4 e_{1}^{2}+s_{1} d_{1}-\left(c_{1} e_{1}+\dot{e}_{1}\right)^{2}-\frac{1}{2 \gamma^{2}} s_{1}^{2} \\
& \leq s_{1} d_{1}-s_{1}^{2}-\frac{1}{2 \gamma^{2}} s_{1}^{2}
\end{aligned}
$$

where 


$$
\left\{\begin{array}{c}
\lambda_{2} \lambda_{1} c_{1}-\lambda_{3}=4 \\
\lambda_{2} \lambda_{3}=1 \\
\frac{c_{1} \lambda_{1}}{\lambda_{2}}-4 \geq 0
\end{array} .\right.
$$

Since $c_{1}$ is a known constant, an empirical value is chosen $\left(c_{1}\right.$ $=2$ ) in this paper. Based on the mathematical relationship of the parameters in Eq. (17), we can calculate $\lambda_{1}=6, \lambda_{2}=0.5, \lambda_{3}$ $=2$.

Secondly, we define the Hamilton function as

$$
\begin{aligned}
H & =\dot{V}_{\text {long }}-\frac{1}{2}\left(\gamma^{2}\left\|d_{1}\right\|^{2}-\left\|z_{1}\right\|^{2}\right) \\
& \leq s_{1} d_{1}-s_{1}^{2}-\frac{1}{2 \gamma^{2}} s_{1}^{2}-\frac{\gamma^{2}}{2}\left\|d_{1}\right\|^{2}+\frac{1}{2}\left\|z_{1}\right\|^{2} \\
& =-\frac{1}{2}\left(\frac{1}{\gamma^{2}} s_{1}^{2}+2 s_{1} d_{1}+\gamma^{2}\left\|d_{1}\right\|^{2}\right)-s_{1}^{2}+\frac{1}{2}\left\|z_{1}\right\|^{2} \\
& =-\frac{1}{2}\left(\frac{1}{\gamma} s_{1}+\gamma d_{1}\right)^{2}-s_{1}^{2}+\frac{1}{2}\left\|s_{1}\right\|^{2} \\
& \leq 0
\end{aligned}
$$

So the $\gamma$-performance criteria are achieved.

$$
\dot{V}_{\text {long }} \leq \frac{1}{2}\left(\gamma^{2}\left\|d_{1}\right\|^{2}-\left\|z_{1}\right\|^{2}\right)
$$

Similarly, the disturbances of vehicle lateral dynamics and the cost function of the lateral control subsystem are given as:

$$
\begin{gathered}
d_{2}(t)=\int_{0}^{t}\left[-a_{2} \frac{v_{y}}{v_{x}}-\left(v_{x}+\frac{k a_{3}}{v_{x}}\right) \dot{\psi} e_{2}\right] \mathrm{d} t \\
z_{2}=s_{2}=c_{2} e_{2}+\dot{e}_{2}
\end{gathered}
$$

where $c_{2}, k_{1}$ and $k_{2}$ are positive constants and

$$
\left\{\begin{array}{l}
e_{2}=k_{1}\left(x_{2}-x_{2, \mathrm{~d}}\right)+k_{2}\left(x_{3}-x_{3, \mathrm{~d}}\right) \\
\dot{e}_{2}=k_{1}\left(\dot{x}_{2}-\dot{x}_{2, \mathrm{~d}}\right)+k_{2}\left(\dot{x}_{3}-\dot{x}_{3, \mathrm{~d}}\right)
\end{array}\right.
$$

Thus, the lateral control law $u_{2}$ has the following form

$$
u_{2}=-\lambda_{1} c_{2} e_{2}-\lambda_{3} \dot{e}_{2}-\frac{1}{2 \gamma^{2}} \frac{I_{z}}{k_{1} I_{z}+k_{2} m l_{f}} s_{2}^{2}+\ddot{x}_{2, \mathrm{~d}}
$$

Notice that the dissipative control laws are formulated to make the IV system with a quadratic dissipative $\gamma$-performance. A suitable $\gamma$ should be determined to produce satisfactory control performance [27]. Generally, we expect a small value of $\gamma$; however, if $\gamma$ is too small it will be liable to cause a large gain of the control system in return. Hence, a step-by-step reduction of the value of $\gamma$ is adopted to optimize the $\gamma$ value. The objective function $J(k)$ is used to restrain the optimization to avoid too small $\gamma$.

$$
J(k)=\sum_{i=1}^{N_{p}} \mathbf{V}(k+i)+\sum_{i=1}^{N_{c}-1}\|\Delta \mathbf{u}(k+i+1)\|^{2}
$$

where $\mathbf{V}=\left[V_{\text {long }}, V_{\text {lat }}\right]^{\mathrm{T}}$ and $\mathbf{u}=\left[u_{1}, u_{2}\right]^{\mathrm{T}}$ denote values of the storage function and control inputs at both longitudinal and lateral directions, respectively; $\Delta \mathbf{u}=\left[\Delta u_{1}, \Delta u_{2}\right]^{\mathrm{T}}$ denote the increments of both longitudinal and lateral control inputs; $k=1$, $2, \ldots, N$ is the iteration step; $N_{p}$ denotes the prediction horizon; $N_{c}$ denotes the control horizon; $\mathbf{V}(k+i)$ represents the value of the storage function at the $k^{\text {th }}$ step in the $i^{\text {th }}$ prediction horizon; $\Delta \mathbf{u}(k+i+1)$ denotes the increments of $\mathbf{u}$ at the $k^{\text {th }}$ step in the $(i+1)^{\text {th }}$ control horizon. During the optimization process, if $\gamma$ still satisfies Eq. (16) at the $k^{\text {th }}$ step but the control inputs $\mathbf{u}$ in the $(i+1)^{\text {th }}$ control horizon at the $k^{\text {th }}$ step are too large, the iteration process will terminate.

\section{VALIDATION}

\section{A. Simulation results}

In order to test the performance of the proposed control laws, different values of $\gamma$ are chosen for successive approximation, and the tracking effect is investigated in the presence of external disturbances. Simultaneously, in terms of the different $\gamma$, the energy-consuming comparison is made for the traction force of the IV. The vehicle and control parameters are shown in Table 2.

As the unknown time varying disturbance of the IV usually causes the limitation of both longitudinal and lateral control inputs, the disturbances $d_{1}$ and $d_{2}$ are restrained to

$$
\left\{\begin{array}{l}
d_{1}=\operatorname{sgn}\left(d_{1}(t)\right) \\
d_{2}=\operatorname{sgn}\left(d_{2}(t)\right)
\end{array}\right.
$$

Simulations last for $30 \mathrm{~s}$, and the simulation step is set to $0.05 \mathrm{~s}$. The initial speed is $25 \mathrm{~m} / \mathrm{s}$, and the IV is running with variable longitudinal and lateral accelerations as shown in Fig. 1. Two different value of $\gamma=1$ (initial value) and $\gamma=0.05$ (optimized value) are chosen to achieve a longitudinal and lateral coupling tracking control for the IV.

TABLE II. VEHICLE AND CONTROL PARAMETERS

\begin{tabular}{cc||cc}
\hline parameters & value & parameters & value \\
\hline $\mathrm{m} / \mathrm{kg}$ & 1640 & $\mathrm{~g} / \mathrm{m} \cdot \mathrm{s}^{-2}$ & 9.8 \\
$I_{z} / \mathrm{kg} \cdot \mathrm{m}^{2}$ & 3105 & $\mu$ & 0.02 \\
$l_{f} / \mathrm{m}$ & 1.193 & $\lambda_{1}$ & 6 \\
$l_{r} / \mathrm{m}$ & 1.587 & $\lambda_{2}$ & 0.5 \\
$C_{f} / \mathrm{N} \cdot \mathrm{rad}^{-1}$ & 131391 & $\lambda_{3}$ & 2 \\
$C_{r} / \mathrm{N} \cdot \mathrm{rad}^{-1}$ & 115669 & $c_{1}, c_{2}$ & 2 \\
$k_{D} / \mathrm{N} \cdot \mathrm{s}^{2} \cdot \mathrm{m}^{-2}$ & 0.49 & $k_{1}$ & 1 \\
$k_{L} / \mathrm{N} \cdot \mathrm{s}^{2} \cdot \mathrm{m}^{-2}$ & 0.008 & $k_{2}$ & 2 \\
\hline
\end{tabular}




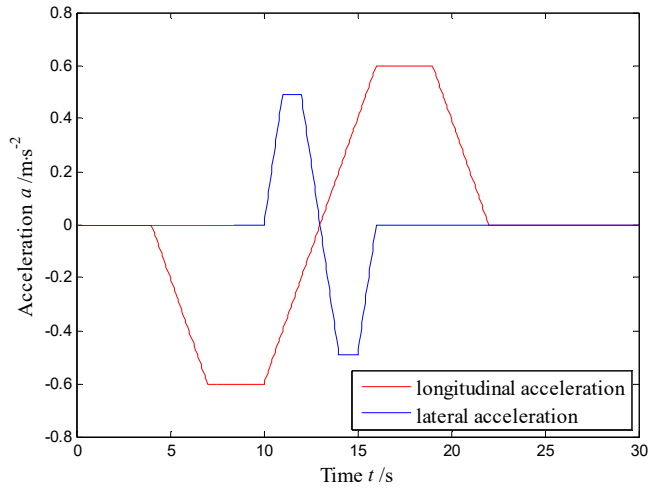

Figure 1. The desired vehicle accelerations.

In the first simulation, the tracking results of the longitudinal, lateral and yaw motion are investigated at both $\gamma$ $=1$ and $\gamma=0.05$, as shown in Figs. 2 and 3.

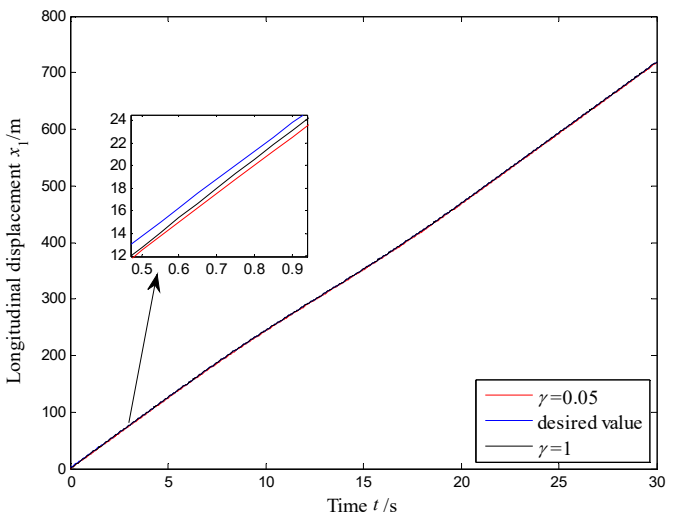

(a) Comparison of the longitudinal displacement

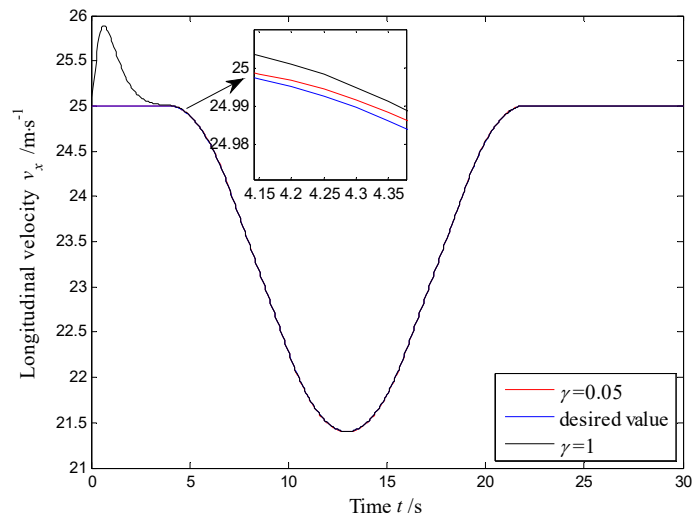

(b) Comparison of the longitudinal velocity

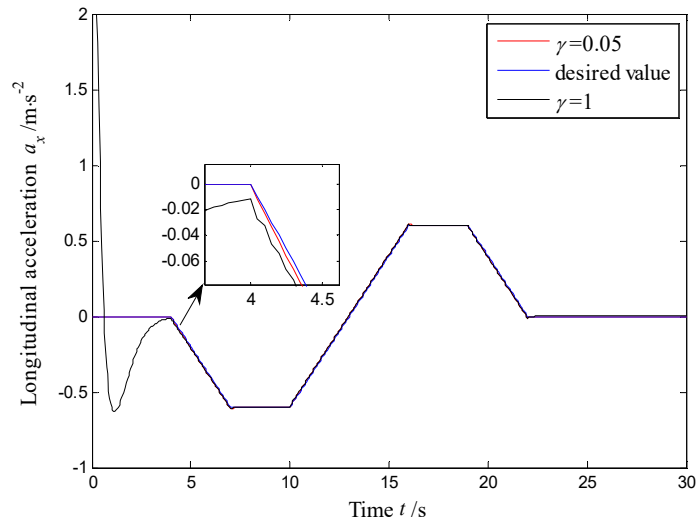

(c) Comparison of the longitudinal acceleration

Figure 2. Longitudinal tracking control simulation comparison.

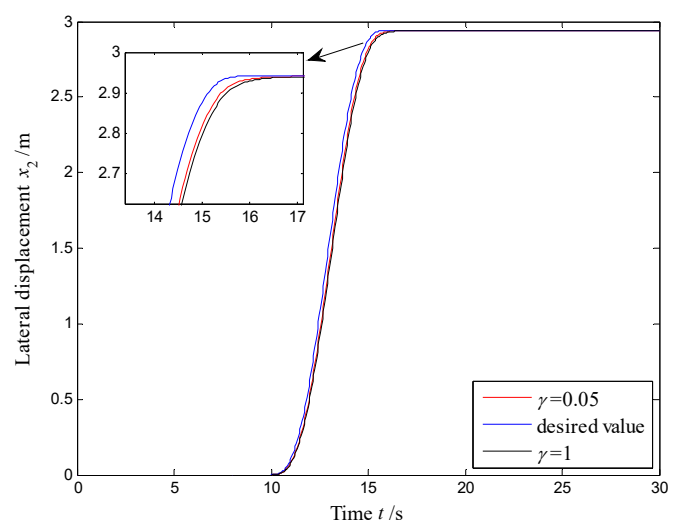

(a) Comparison of the lateral displacement.

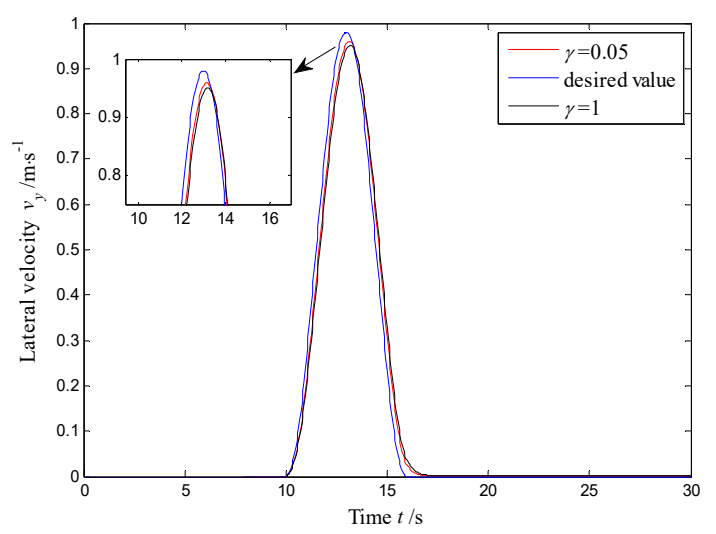

(b) Comparison of the lateral velocity. 


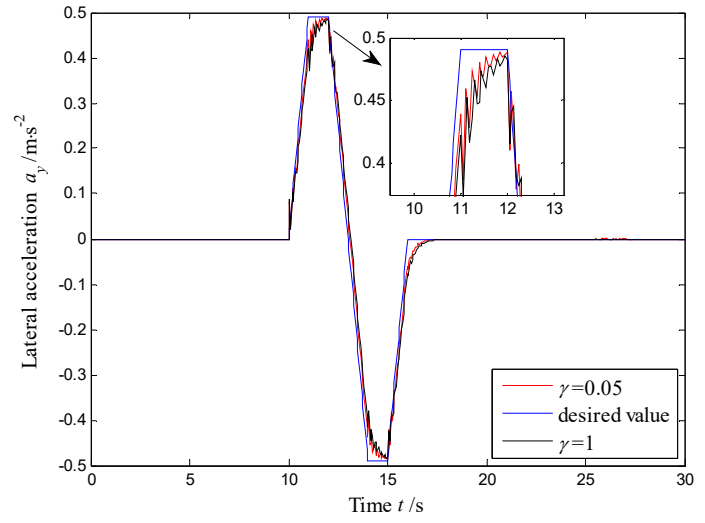

(c) Comparison of the lateral acceleration.

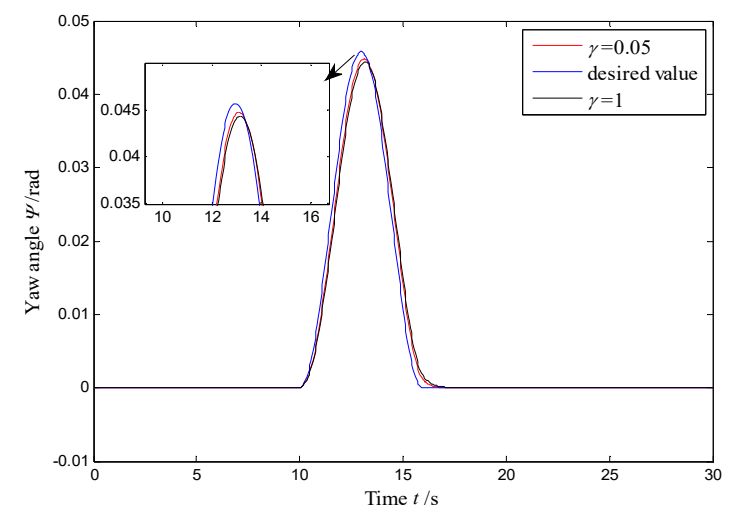

(d) Comparison of the yaw angle.

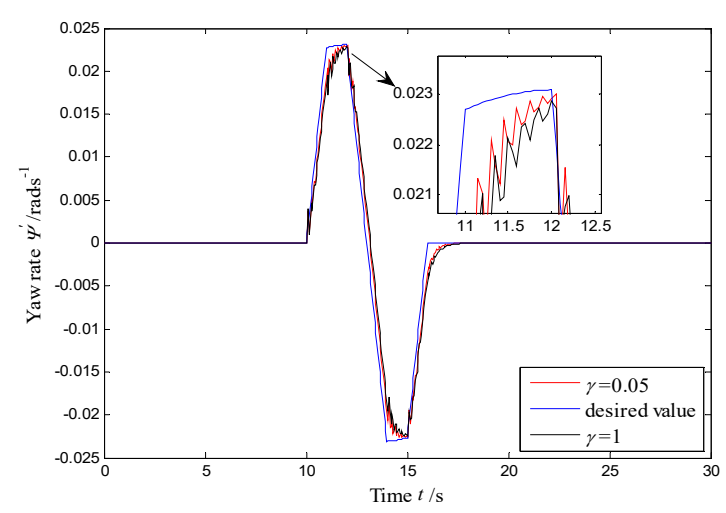

(e) Comparison of the yaw rate.

Figure 3. Lateral tracking control simulation comparison.

As can be seen from Figs. 2 and 3, the tracking accuracy of the control system can be increased quickly by gradually reducing the value of $\gamma$, but if the value of $\gamma$ is too small, the larger gain of the control system will appear and result in a clear chattering. Additionally, it is obvious that the longitudinal tracking control has less influence on the parameter uncertainties and external disturbance than the lateral tracking control. It appears that the structure of the lateral storage function needs to be improved with regards to the choice of the weights, integrators and feedback based on errors measured at the c.o.g. leads to bad ride comfort.

The second simulation shows the energy consumption comparison of the throttle/brake inputs at $\gamma=1$ and $\gamma=0.05$, respectively. In general, the amount of energy consumption per unit time is proportional to the work that both vehicle traction force and braking force do. Fig. 4 shows a comparison of the regulation of the traction force at the two different values of $\gamma$.

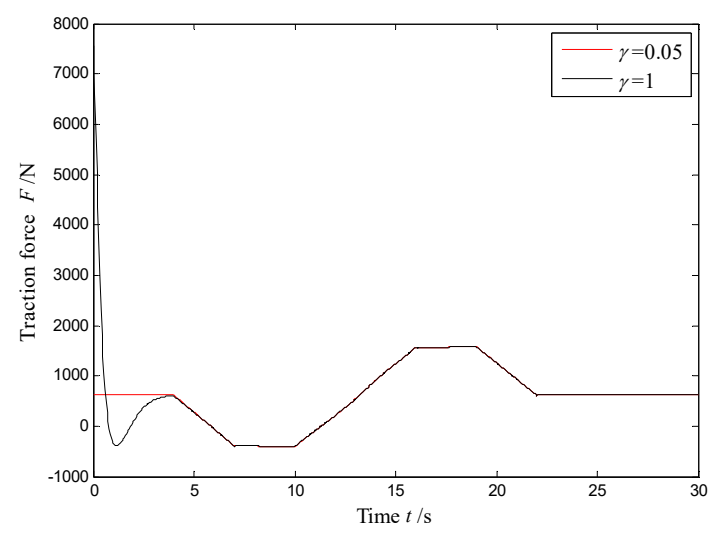

Figure 4. Comparison of the regulation of traction force.

As both the throttle and brake dynamics are discounted in this paper, and the control inputs are directly exerted onto the vehicle system, parts of the traction force thus have the negative values depicted in Fig. 4. According to the interactions between the traction force and the braking force, it should be noticed that, when the traction force is negative, it is identical to what the braking force works. Therefore, the energy consumption in the whole simulation is simplified by the calculation of $\Sigma|F|$. This way, the comparison of the energy consumption at the two supply rates is as shown in Fig. 5.

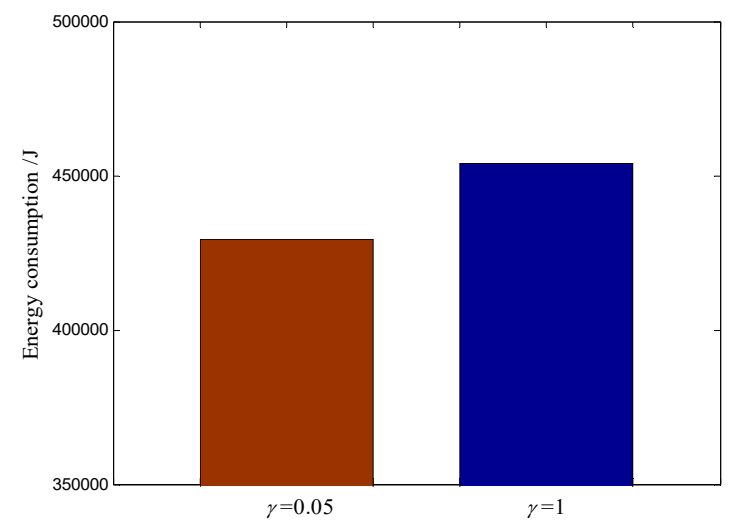

Figure 5. Comparison of the energy consumption.

It can be seen from Fig. 5, compared with 453,820 J at $\gamma=1$, the IV only consumes a total energy of $429,380 \mathrm{~J}$ at $\gamma=0.05$, i.e., around $5 \%$ reduction in the energy consumption rate. This 
shows the dissipative feedback control law does reduce the energy consumption.

The third simulation shows the adaptive speed control comparison of two separate control strategies under the slope road, constant speed driving (CSD) and adaptive speed control driving (ASCD). CSD uses the PID control algorithm and ASCD uses the proposed dissipative control algorithm with $\gamma=$ 0.05 . The slope road is given in Fig. 6 .

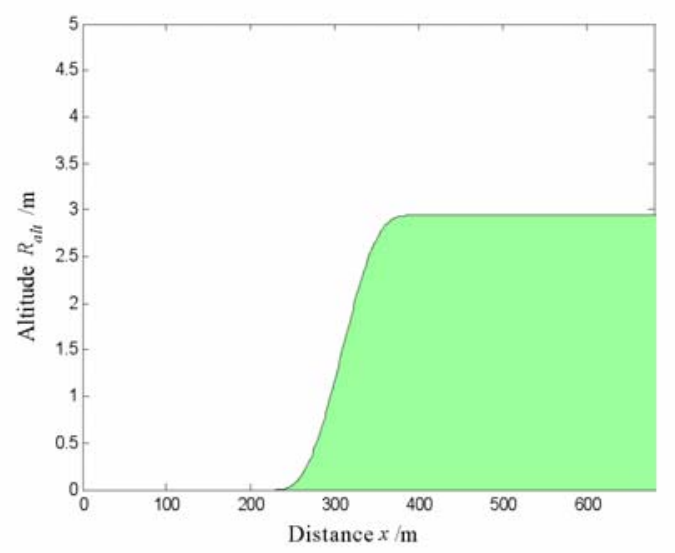

(a) Slope road

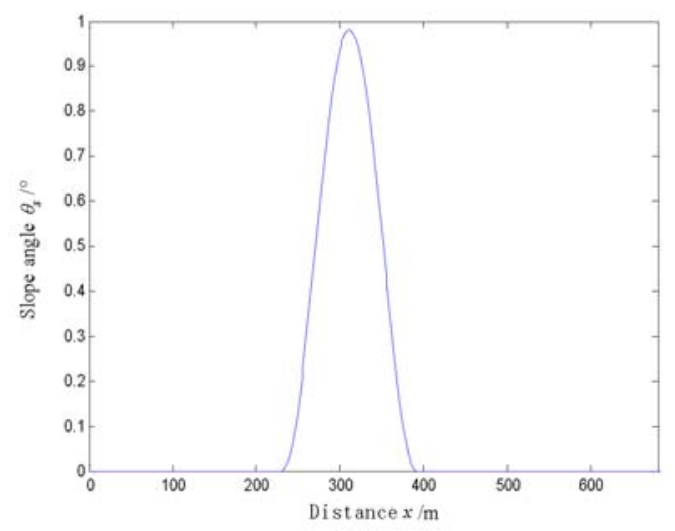

(b) Slope angle

Figure 6. The simulation of slope road.

The adaptive speed control simulation is performed for this slope scenario. Assuming the initial velocity is $22 \mathrm{~m} / \mathrm{s}$, ASCD and CSD are employed in a comparative analysis on the velocity, traction force and energy consumption. As the longitudinal acceleration is influenced by the gravity components caused by the road slope angle, the external disturbance has the following form

$$
d=\ddot{x}_{1}+g \sin \theta_{s}
$$

It can be seen from Fig. 7(a) that the ASCD vehicle can reach the top of the slope in an adaptive way with an operation profile of "pre-accelerating-post-decelerating." Despite that the speed profile of the ASCD vehicle shows a short-term increase and decrease in the early stage (i.e., before reaching the slope), possibly due to some issue in the computation of the cost function, the profile has a good, smooth shape over the entire slope. In contrast, the CSD vehicle keeps the velocity unchanged at the cost of a higher traction forces, easily consuming more energy and making the actuators in more danger.

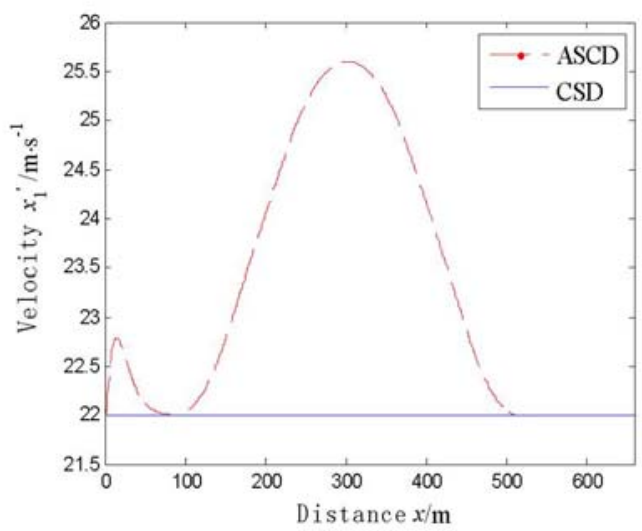

(a) Comparison of the velocity

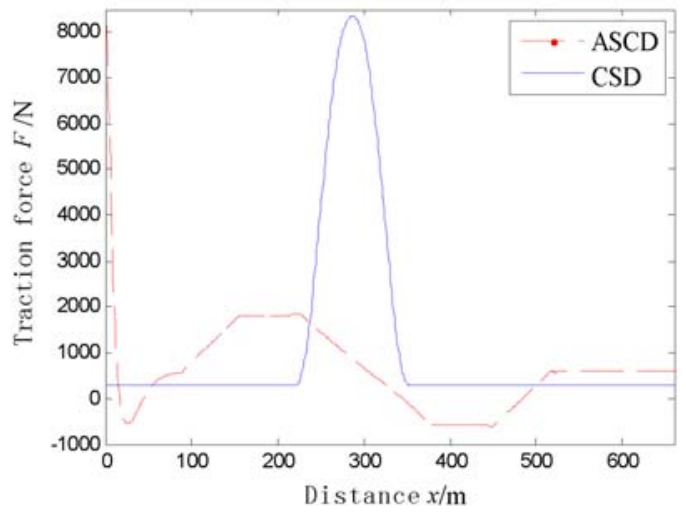

(b) Comparison of the traction force

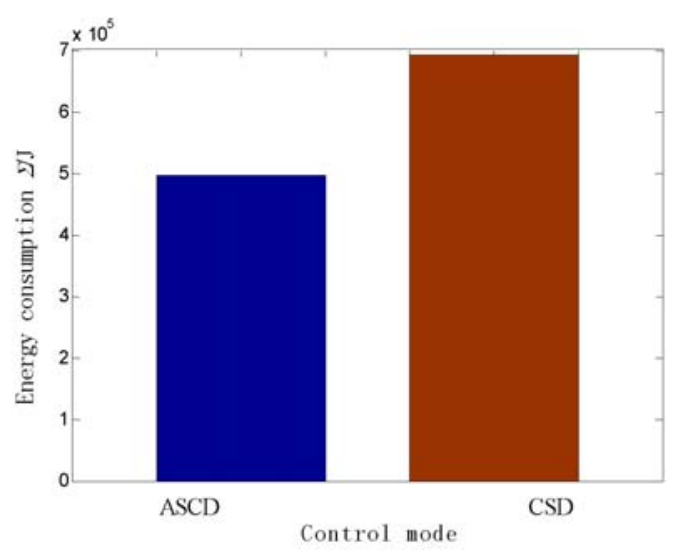

(c) Comparison of the energy consumption

Figure 7. The adaptive speed control simulation under slope scenario.

Fig. 7(b) shows the traction/braking force profiles under two types of speed control for the slope scenario. The force of 
the CSD vehicle changes greatly when the vehicle runs on the slope, while the force of the ASCD vehicle vary slightly over the slope. In addition, the performance of the two types of speed controllers is compared from the perspective of the energy consumption, as shown in Fig. 7(c), the total force of the CSD vehicle is $692,280 \mathrm{~N}$, while that for the ASCD vehicle is $496,300 \mathrm{~N}$. So the energy consumption of the proposed method is much smaller than that of the PID. As a result, it can be concluded that under the same slope and initial speed condition, the proposed ASCD method in this paper can reduce the consumption of the control energy by $28.3 \%$ compared to the PID controller.

\section{B. Testbed results}

In the testbed experiment, a scaled IV, driving along a curved and sloped road is described, as shown in Fig. 8. The scaled vehicle is equipped with a digital-signal-processor (DSP), power supply module, motor drive module that controls the steering motor and the driving motor, and several on-board sensors, including a digital camera for image recognition of the surface, and an optical encoder for vehicle motion acquisition.

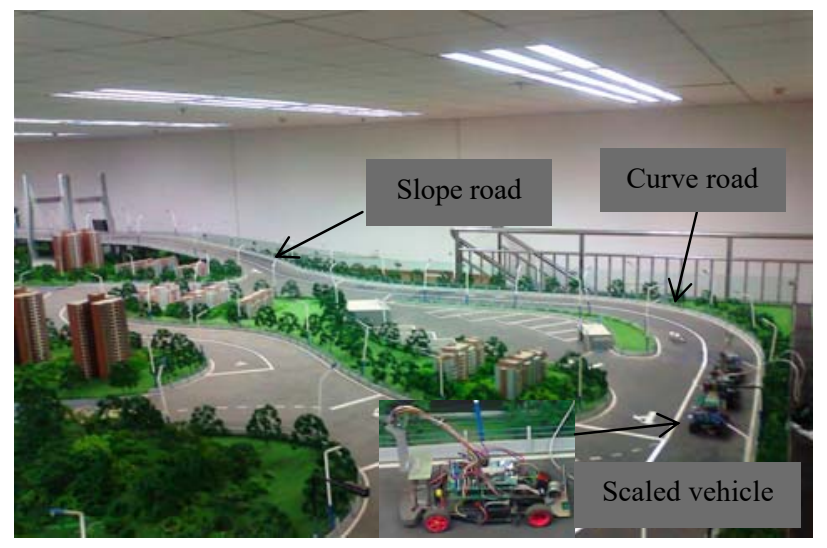

Figure 8. Test-bed experiment setup.

The sloped road is approximately $1000 \mathrm{~cm}$ long and its height is approximately $40 \mathrm{~cm}$. The external disturbance is mainly manifested at the slope angle, and it has an immediate impact on the change of the vehicle's longitudinal acceleration. For the up slope in Fig. 8, the longitudinal acceleration is influenced by the gravity components caused by the road slope angle. The curved road is approximately $400 \mathrm{~cm}$ long and its maximum radius is $125 \mathrm{~cm}$. The external disturbance is mainly manifested in the road curvature, and it also has an immediate impact on the change of the vehicle's lateral acceleration. For the curve in Fig. 8, the lateral acceleration is mainly influenced by the desired yaw rate caused by the road curvature. Thus, the disturbances in these driving scenes are defined as:

$$
\left\{\begin{array}{c}
d_{1}=\ddot{x}_{1}+g \sin \theta_{s} \\
d_{2}=\ddot{x}_{2}+d_{s}\left(\ddot{x}_{3}-C \dot{x}_{1}\right)
\end{array}\right.
$$

where $d_{s}$ is the horizontal distance to the sensor from the vehicle center of gravity (c.o.g), and $\theta_{s}$ and $C$ are the road slope angle and curvature, respectively. The profiles of $\theta_{s}$ and $C$ over the driving distance are measured and shown in Fig. 9.

The adaptive speed control experiment is performed under both slope and curve scenarios, assuming the scaled vehicle enters these driving scenarios at an initial speed of $1 \mathrm{~m} / \mathrm{s}$. ASCD and CSD are employed in a comparative analysis on the velocity.

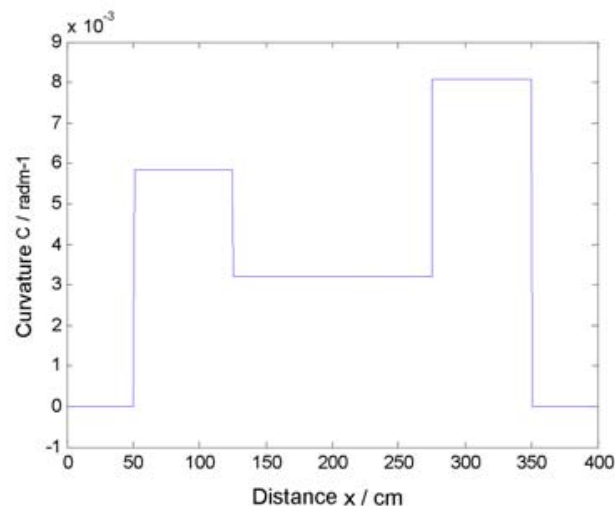

(a) Road curvature.

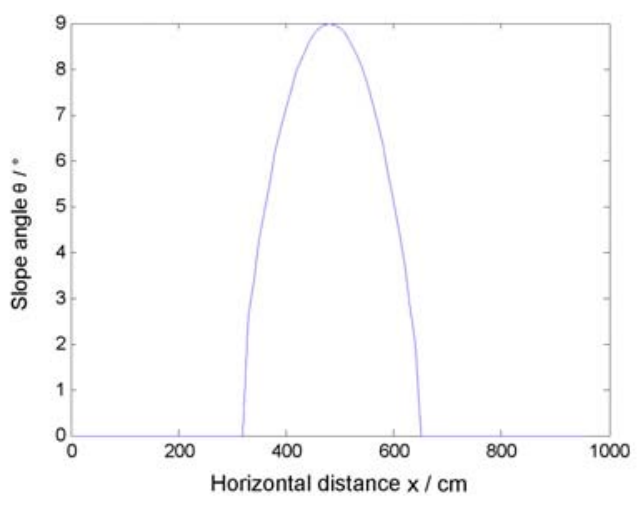

(b) Slope angle.

Figure 9. Road geometrical features.

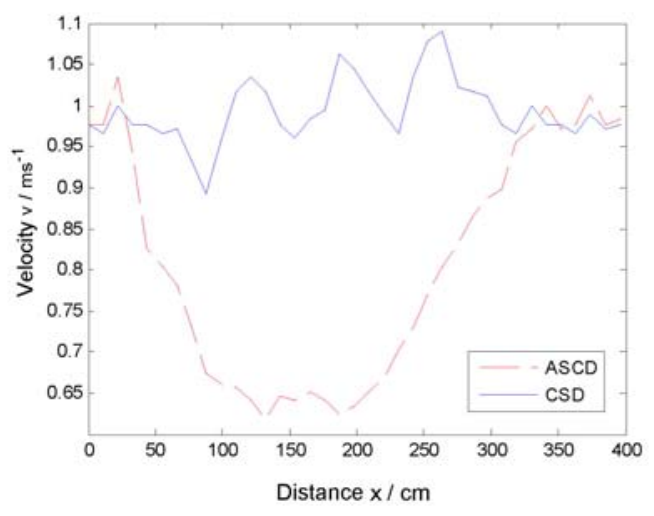

(a) In the curve. 


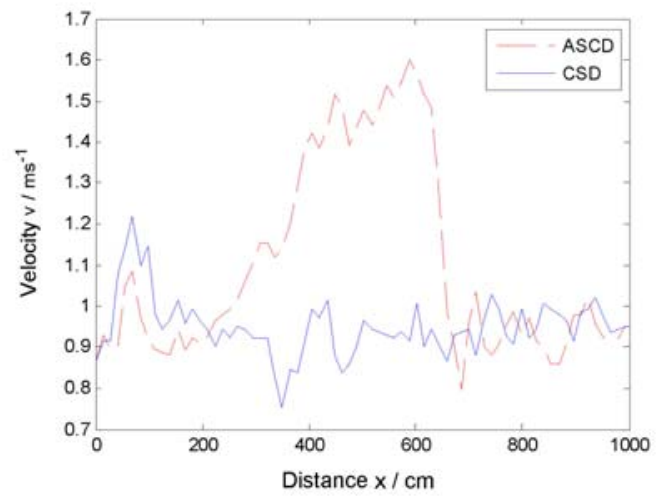

(b) In the up slope.

Figure 10. Comparison of the actual velocity in different road geometry.

It can be seen from Fig. 10(a) that the CSD vehicle passes the curve at a constant velocity, unchanged with the curvature. The ASCD vehicle, on the other hand, can regulate the velocity following in line with the curvature. In order to ensure driving safety, the ASCD vehicle decelerates the velocity when approaching the curve and it accelerates before driving out of the curve, which achieves automated speed adaptation and reduces the frequent chattering arising from the greater amount produced in the control process.

It can be seen from Fig. 10(b) that the CSD vehicle passes the up slope at a constant velocity, unchanged with the slope angle. The ASCD vehicle, on the other hand, can regulate the velocity following in line with the slope. In order to improve the mobility performance, the ASCD vehicle accelerates when approaching the slope and decelerates the velocity when nearing the top of the slope. This also achieves an automated speed adaptation and can reduce the frequent chattering arising from the greater amount produced in the control process.

Finally, it is observed that there is a small velocity fluctuation when the ASCD vehicle is going to enter the curve or slope road, because the first term of the storage functions (13) constitutes a larger proportion of the dissipative control law calculation, the unsuitable term might lead to an obvious overshoot in the change of the vehicle's velocity. Although the overshoot appears small in the figures, the storage functions need be well developed to ensure driving smoothness in more severe cases, such as steep slopes, sharp curves, etc.

\section{V.CONCLUSION}

This paper has proposed a new dissipative control approach based on energy storage function. A back-stepping based Lyapunov method is used to develop a storage function satisfying the $\gamma$-performance with respect to the quadratic supply rate, and a dissipative control law is obtained by gradually reducing the value of $\gamma$, whose stability is achieved in the adaptive speed control simulations in the presence external disturbances and slope road. Also, results from the adaptive speed control experiments under both curve and slope road conditions suggest better driving safety and comfort by adapting to the changing road geometry. From the comparison results between the proposed new method and a simple controller (i.e., PID controller) it is observed significant improvement on the control performance using the proposed dissipative control method. Since our method does not require solving Riccati equations or HJI inequalities, it does not need offline computation and can be applied directly in on-line usage. That means the proposed method is simple in implement although it has relatively complex control design process compared with PID. Hence, the propose method contributes a good balance between control precision and simplicity. Future work will focus on further investigating the expressions of the parameter uncertainties and external disturbance, and considering the actuators of the brake, throttle, and steering dynamics, all of which may help further improve the driving safety, ride comfort and fuel economy of the IV. Moreover, we plan to conduct a comprehensive investigation to compare the energy dissipation based method with platoon control techniques in terms of computational efficiency.

\section{REFERENCES}

[1] D. González, J. Pérez, V. Milanés, and F. Nashashibi, "A Review of Motion Planning Techniques for Automated Vehicles," IEEE Transactions on Intelligent Transportation Systems, vol. 17, no. 4, pp. 1135-1145, 2016

[2] H. Li, F. Nashashibi, and M. Yang, "The Split Covariance Intersection Filter: Theory, and Its Application to Vehicle Localization," IEEE Transactions on Intelligent Transportation Systems, vol. 14, no. 4, pp.1860-1871, 2013

[3] D. González, J. Pérez, V. Milanés, and F. Nashashibi, "A review of motion planning techniques for automated vehicles," IEEE Transactions on Intelligent Transportation Systems, vol. 17, no. 4, pp.1135-45, 2016

[4] J. Pérez, V. Milanés, and M. Penas, "Control agents for autonomous vehicles in urban and highways scenarios." IFAC Proceedings, vol. 46, no. 10 , pp. $120-125,2013$

[5] J. Pérez, V. Milanés, J. Godoy, J. Villagra, and E. Onieva, "Cooperative controllers for highways based on human experience," Expert Systems with Applications, vol. 40, no. 4, pp.1024-1033, 2013

[6] K. Santhanakrishnan and R. Rajamani. "On Spacing Policies for Highway Vehicle Automation," IEEE Transactions on Intelligent Transportation Systems, vol. 4, no. 4, pp. 198-204, 2003

[7] X.Y. Lu, and J.Q. Wang. "Multiple-Vehicle Longitudinal Collision Avoidance and Impact Mitigation by Active Brake Control," in Proceedings of IEEE Intelligent Vehicles Symposium, pp. 680-685, Alcalá de Henares, Spain, June, 2012

[8] D.Z. Zhang, K.Q. Li, and J.Q. Wang. "A Curving ACC System with Coordination Control of Longitudinal Car-following and Lateral Stability," Vehicle System Dynamics, vol. 50, no. 7, pp. 1085-1102, 2012

[9] J. Piao, and M. McDonald, "Advanced Driver Assistance Systems from Autonomous to Cooperative Approach," Transport Reviews, vol. 28, no. 5, pp. 659-684, 2008

[10] Guo G, Yue W. Autonomous platoon control allowing range-limited sensors. IEEE Transactions on vehicular technology, 2012, 61(7): 2901-2912.

[11] Guo G, Yue W. Sampled-data cooperative adaptive cruise control of vehicles with sensor failures. IEEE Transactions on Intelligent Transportation Systems, 2014, 15(6): 2404-2418.

[12] Li Shengbo Eben, Gao Feng, Cao Dongpu, et al. Multiple-model switching control of vehicle longitudinal dynamics for platoon level automation. IEEE Transactions on Vehicular Technology, 2016, 65(6):4480-4492. 
[13] Qin Xia, Feng Gao, Jianli Duan, et al. Decoupled H-inf Control of Automated Vehicular Platoons with Complex Interaction Topologies. IET Intelligent Transport Systems, 2017, 11(2):92-101

[14] R. Garcia, T. de Pedro, J. Naranjo, J. Reviejo, and C. Gonzalez, "Frontal and lateral control for unmanned vehicles in urban tracks," In Proceedings of Intelligent Vehicle Symposium, pp. 3118-3125, Versailles, France, June 2002

[15] S. Hosseinnia, I. Tejado, V. Milanés, J. Villagrá, and B. Vinagre, "Experimental application of hybrid fractional-order adaptive cruise control at low speed," IEEE Transactions on Control Systems Technology, vol. 22, no. 6, pp. 2329-2336, 2014

[16] E. Onieva, J. Naranjo, V. Milanés, J. Alonso, R. García, and J. Pérez, "Automatic lateral control for unmanned vehicles via genetic algorithms," Applied Soft Computing, vol. 11, no. 1, pp. 1303-1309, 2011

[17] J. Zhang and P. Ioannou, "Longitudinal control of heavy trucks in mixed traffic: environmental and fuel economy considerations," IEEE Transactions on Intelligent Transportation Systems, vol. 7, no. 1, pp. 92-104, 2006

[18] D. Corona, M. Lazar, B. De Schutter, and M. Heemels, "A hybrid MPC approach to the design of a Smart adaptive cruise controller," in Proceedings of the 2006 IEEE International Conference on Control Applications, Munich, Germany, pp. 231-236, Oct 2006

[19] S. Velhal and S. Thomas, "Improved LTVMPC design for steering control of autonomous vehicle," Journal of Physics: Conference Series, vol. 783, no. 1, pp. 012028, 2017

[20] A. Katriniok, J. Maschuw, F. Christen, L. Eckstein, and D. Abel, "Optimal vehicle dynamics control for combined longitudinal and lateral autonomous vehicle guidance," In Proceedings of European Control Conference, pp. 974-979, Zürich, Switzerland, July 2013

[21] R. Kianfar, M. Ali, P. Falcone, and J. Fredriksson, "Combined longitudinal and lateral control design for string stable vehicle platooning within a designated lane," In Proceedings of Intelligent Transportation Systems (ITSC), pp. 1003-1008, Qingdao, China, Oct 2014

[22] R. Attia, R. Orjuela, and M. Basset, "Combined longitudinal and lateral control for automated vehicle guidance," Vehicle System Dynamics, vol. 52, no. 2, pp. 261-279, 2014

[23] X. Yan, R. Zhang, J. Ma, and Y. Ma. "Considering variable road geometry in adaptive vehicle speed control," Mathematical Problems in Engineering, vol. 2013, pp. 1-12, 2013

[24] Y. Ma, Q. Wu, X. Yan, and R. Zhang, "The hardware-in-the-loop simulator: A mechatronic testbed for cooperative vehicles maneuvers," International Journal of Intelligent Transportation Systems Research, vol. 11, no. 1, pp. 11-22, 2013

[25] Y. Yamamura, Y. Seto, H. Nishira and T. Kawabe. "An ACC Design Method for Achieving Both String Stability and Ride Comfort," Journal of System Design and Dynamics, vol. 2, no. 4, pp. 979-990, 2008

[26] S. Yuliar, M. R. James, and J. W. Helton. "Dissipative control systems synthesis with full state feedback," Mathematics of Control, Signals and Systems, vol. 11, no. 4, pp. 335-356, 1998

[27] J. C. Willems. "Dissipative dynamical systems part I: General theory, Part II: Linear systems with quadratic supply rates," Archive for Rational Mechanics and Analysis, vol. 45, no. 5, pp. 352-393, 1972

[28] J.C. Willems. "Lyapunov functions for systems described by high order differential equations," in Proceedings of the 30th IEEE Conference on Decision and Control, pp. 900-904, Brighton, England, December 1991

[29] A. J. van der Schaft, " $L_{2}$ gain analysis of nonlinear systems and nonlinear state feedback $H_{\infty}$ control," IEEE Transactions on Automatic Control, vol. 37, no. 6, pp. 770-784, 1992.

[30] L. Nehaoua and L. Nouvelière, "Backstepping based approach for the combined longitudinal-lateral vehicle control," in Proceedings of IEEE Intelligent Vehicles Symposium, pp. 395-400, Alcalá de Henares, Spain, June, 2012

[31] Ya-Fu Peng, "Adaptive intelligent backstepping longitudinal control of vehicle platoons using output recurrent cerebellar model articulation controller," Expert Systems with Applications, vol. 37, pp. 2016-2027, 2010
[32] D.B. Ren, J.Y. Zhang, J.M. Zhang, and S.M. Cui. "Trajectory planning and yaw rate tracking control for lane changing of intelligent vehicle on curved road," Sci China Tech Sci, vol. 41, no. 3, pp. 630-642, 2011.

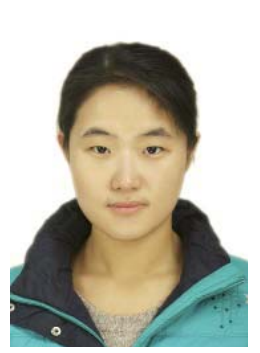

Dr. Rui Zhang received her $\mathrm{PhD}$ in Transportation Engineering from Wuhan University of Technology, China. Currently she is a lecturer with the School of Automotive and Transportation, Tianjin University of Technology and Education, China. Her main research interests include intelligent transportation systems and

intelligent vehicles.

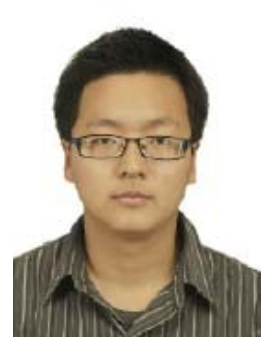

Dr. Yulin Ma (M'13) received his PhD in Transportation Engineering from Wuhan University of Technology, China. He was a Post Doctor with Academy of Military Transportation, China. Currently he is an Associate Professor with National Center of ITS Engineering and Technology, Research Institute of Highway, Ministry of Transport, China. His research interests include intelligent vehicles and intelligent transportation systems. He is currently a reviewer for the IEEE Transactions on Intelligent Transportation Systems, International Journal of Intelligent Transportation Systems Research.

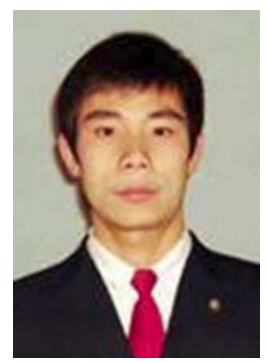

Dr. Zhixiong Li (M'16) received his $\mathrm{PhD}$ in Transportation Engineering from Wuhan University of Technology, China. Currently he is a Senior Lecture with China University of Mining and Technology, China, and a research associate in Department of Mechanical Engineering, Iowa State University, USA. His research interests include mechanical system modeling and control. He is an associate editor for the Journal of IEEE Access.

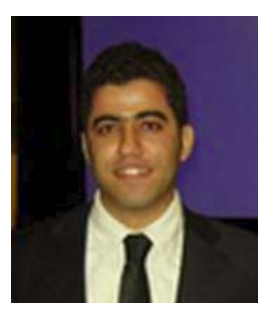

Dr. Reza Malekian (M'12) is currently a Senior Lecturer with the Department of Electrical, Electronic, and Computer Engineering, University of Pretoria, Pretoria, South Africa. His current research interests include advanced sensor networks, Internet of Things, and mobile communications. Dr. Malekian is also a Chartered Engineer and a Professional Member of the British Computer Society. He is an associate editor for the Journal of IEEE Internet of Things. 


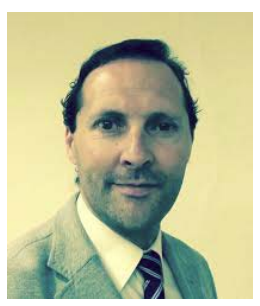

Professor Miguel Angel Sotelo received his Ph.D. degree in Electrical Engineering in 2001 from the University of Alcalá (UAH), Alcalá de Henares, Madrid, Spain. He is Head of the INVETT Research Group and Vice-President for International Relations at the University of Alcalá. He has been the Editor-in-Chief of IEEE Intelligent Transportation Systems Magazine, (2014-2016) and an Associate Editor of IEEE Transactions on Intelligent Transportation system (2008-2015). Currently, he is the President-Elect of the IEEE Intelligent Transportation Systems Society (starting Jan 2017). 